\title{
Amperometric Readout and Electrode Array Chip for Bioelectrochemical Sensors
}

\author{
Andrew Mason, Yue Huang, Chao Yang and Jichun Zhang \\ Electrical and Computer Engineering, Michigan State University, E. Lansing MI, USA
}

\begin{abstract}
As nanostructured bioelectronic interfaces continue to evolve for sensor applications, new readout circuits are needed to harness their capabilities. This paper presents a single-chip amperometric readout circuit and electrode array system suitable for bioelectrochemical measurements. The chip features a CMOS potentiostat with high resolution, rangeprogrammable current readout and electrochemical cell potential drive circuitry, which can perform on-chip chronoamperometry and cyclic voltammetry assays. Through post-CMOS fabrication, the surface of the chip is prepared with an array of electrodes suitable for formation of bioelectronic interfaces and on-chip bioelectrochemical measurements. The $3 \times 3 \mathrm{~mm}^{2}$ chip nominally hosts a $4 \times 4$ working electrode array and supports amperometric outputs ranging from $10 \mathrm{pA}$ to $10 \mu \mathrm{A}$ with sub-pA resolution.
\end{abstract}

\section{INTRODUCTION}

Measuring analyte concentrations is essential for the study of biological systems and the development of tools for disease diagnosis and treatment. Electrochemical biosensors have been widely used to determine analyte concentrations, both in research and commercial applications [1]. Bioelectrochemical sensors can detect protein activity either directly [2] or indirectly through reaction coupling $[3,4]$ to greatly broaden the range of protein classes that can be used as biological recognition elements (BRE). Although optical measurement techniques are prevalent in biosensors today, BREs with electrically coupled outputs have several advantages including, less/no sample preparation required, elimination of performance-limiting optical interference, and suitability for chip-scale integration of entire analysis platforms. However, breakthroughs are needed to permit cost-effective implementation of high performance bioelectrochemical sensor arrays.

Electrochemical BREs utilize three-electrode electrochemical measurement techniques and interface with electronics through a potentiostat, which can be configured for potentiometric (apply current and measure output voltage) or amperometric (apply voltage and measure output current) readout. As previously cited [5], several integrated circuit potentiostats have been developed. However high density electrochemical sensor arrays based on nanostructured bioelectronic interfaces [6] present several new constraints that must be addressed. To exploit the sensitivity of proteinbased BREs, the readout electronics must have high resolution and suppress noise. To accommodate the variable response of

This work was partially supported by the Michigan Economic Development Corp. under the Technology Tri-Corridor Fund and the NSF Center for Wireless Integrated Microsystems (WIMS) under award number ERC-9986866. a diversity of BREs, the interface circuit must support a large signal range. For amperometric detection, which is preferred for most bioelectronic interfaces, these requirements translate to current sensitivities ranging from $1 \mathrm{fA}$ to $1 \mathrm{nA}$ as full-scale output spans from $10 \mathrm{pA}$ to $10 \mu \mathrm{A}$. Thus, a low-level current detection circuit with high linearity and strong noise performance is required. High resolution potentiostats have been demonstrated using charge integrators that convert currents to voltages or digital pulses [7-10]. However, their range of permissible input currents and supported interrogation techniques are not well matched to protein-based sensors. In contrast, this paper reports a single-chip platform suited to formation and measurement of bioelectrochemical sensor arrays. The highly sensitive readout circuit can measure a wide range of currents from an array of electrodes patterned on the chip using post-CMOS processing, permitting amperometric characterization of a diversity of proteins.

\section{CHIP ARCHITECTURE AND OPERATION}

Fig. 1 shows a 3D conceptual illustration and block diagram of circuit functions for the bioelectrochemical array microsystem. A reference electrode (RE), a counter electrode (CE), and a $4 \times 4$ array of working electrodes (WE) are patterned on the surface of an electrochemical interface circuit using post-CMOS fabrication. The amperometric readout block and the electrode drive block combine to realize electrochemical potentiostat functions. The electrode drive block controls the potential across a specific electrochemical cell that is selected by a current switch matrix at the input of the readout block, and the chosen current is integrated, amplified and fed to an $\mathrm{A} / \mathrm{D}$ converter. The electrode potential drive block is configured to support either static voltage (chronoamperometry) or cyclic voltammetry (CV) assays, with digital signals controlling a DAC that establishes the applied potential. The circuit can operate with either an onchip or external reference electrode, and the generated potential at the RE is output from the chip to assist in plotting $\mathrm{CV}$ curves. The readout circuits are required to provide low noise, high resolution measurements with high linearity over a wide current range suitable for various transducers and electrode sizes. The electrodes are required to be compatible with post-CMOS processing and to maintain a reliable interface with subsequently applied biological materials.

A simplified schematic of the three-electrode bioelectrochemical interface chip is shown in Fig. 2. In the electrode potential drive block, control signal Vsrc sets the potential applied across the electrochemical cell, and either a constant voltage or a sweeping signal for $\mathrm{CV}$ measurements can be applied. Amp2 is configured as a unity gain buffer to 


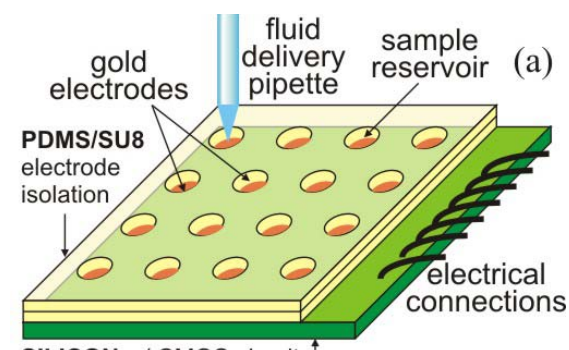

SILICON w/ CMOS circuits $\uparrow$

\begin{tabular}{|c|c|c|}
\hline \multicolumn{2}{|c|}{ surface electrode array } & \multirow[t]{2}{*}{ (b) } \\
\hline WE array & CE RE & \\
\hline $\begin{array}{c}\text { amperometric } \\
\text { readout }\end{array}$ & $\begin{array}{c}\text { electrode } \\
\text { drive }\end{array}$ & \multirow{2}{*}{$\begin{array}{c}\text { digital } \\
\text { comm } \\
\& \\
\text { control }\end{array}$} \\
\hline$\frac{1}{A / D}$ & DA & \\
\hline
\end{tabular}

Figure 1. Conceptual illustration of the electrochemical array system on chip; (a) top view, (b) functional circuit diagram.

establish a potential difference between the RE and the chosen WE. Amp1 and Amp2 form a feedback circuit to control the $\mathrm{RE}$ potential through the $\mathrm{CE}$. Notice that the RE can not sink/source electrical current, so all current measured at the WE comes from the CE, which helps maintain stability of the RE electrochemical potential.

In the current readout block [5], a switched capacitor (SC) charge integrator converts the sensor output current into a voltage, which then goes through a programmable gain amplifier (PGA) with auto-zero compensation. The output voltage is then sampled, held, and fed to an $\mathrm{A} / \mathrm{D}$ converter.

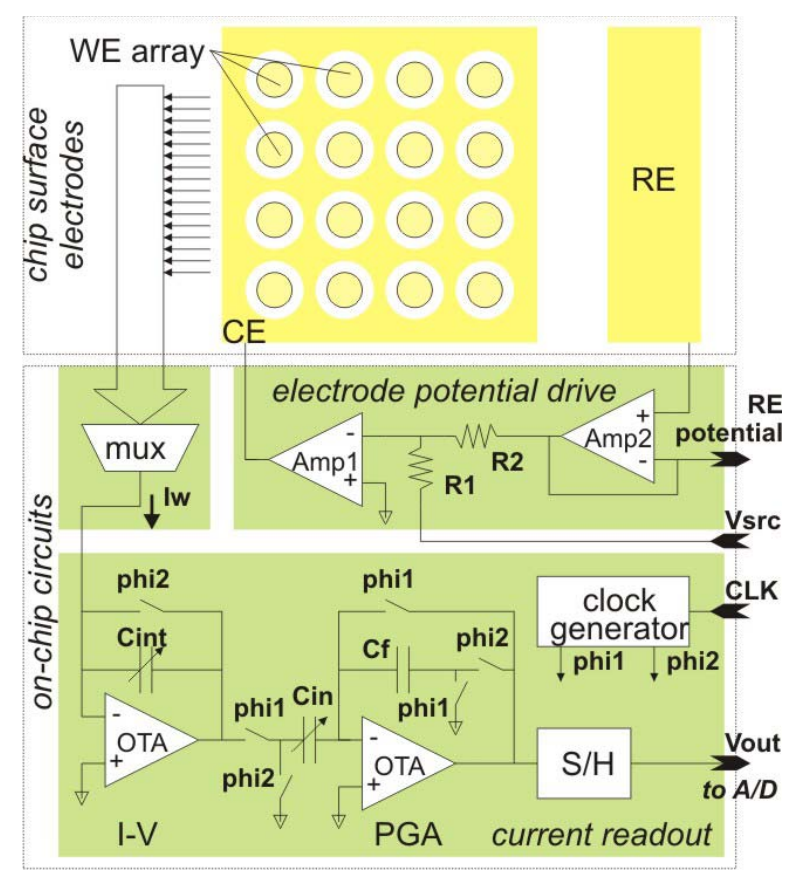

Figure 2. Schematic of the surface electrode array and the on-chip potentiostat comprised of a switched capaitor amperometric readout block, an electrode potential drive block, and a current switching matrix.
The entire readout chain utilizes correlated double sampling (CDS) [11] to reduce the 1/f noise of the PGA and compensate for amplifier offset. The output of the highly sensitive current readout block, $V_{\text {out }}$, is given by [5]:

$$
V_{\text {out }}=\frac{I_{w}}{C_{\text {int }} f_{s}} \cdot \frac{C_{\text {in }}}{C_{f}}
$$

where, $f_{s}$ is the frequency of phil, $I_{w}$ is the sensor current from the working electrode, $C_{i n t}$ is the integrator capacitor, $C_{i n}$ is the binary weighted programmable capacitor at the PGA input, and $C_{f}$ is the feedback capacitor of the PGA.

The response sensitivity of bio-interfaces applied to the WE array can vary widely, resulting in a broad range of currents to be measured by the readout circuit. The current generated by the transducer is also directly proportional to the area of the WE, which can readily be patterned to any diameter between $\sim 10 \mu \mathrm{m}$ and $2 \mathrm{~mm}$, depending on the application. To support these variable current levels, which are not set until after the electrochemical interface circuit is fabricated, it is vital that the current readout block operate over a wide range of input currents. As shown by (1), the overall readout circuit gain $\left(V_{\text {out }} / I_{w}\right)$ is determined by the clock frequency, the PGA gain $\left(C_{i n} / C_{f}\right)$, and the value of the integrator capacitor. Thus, by using on-chip programmable capacitors for $C_{i n t}$ and $C_{i n}$ and adjusting the clock frequency, the readout circuit can be adapted to a large input current span. The capacitor values and operational frequency were designed to support input currents ranging from $10 \mathrm{pA}$ to $10 \mu \mathrm{A}$.

To achieve high sensitivity in the current readout circuit, care has been taken to minimize the noise power due to the amplifiers. With the CDS technique, low frequency $1 / \mathrm{f}$ noise can be sampled and subtracted. Since speed is not critical limitation, relatively large capacitors have been implemented to control $\mathrm{kT} / \mathrm{C}$ noise. As a result, noise is suppressed significantly at the output. Most of the switches are controlled by a charge injection insensitive clock scheme. Other switches use minimum sized CMOS transmission gates to minimize the charge injection, and dummy switches have also been used to reduce clock feedthrough errors in the amplifiers.

\section{POST-CMOS ARRAY FABRICATION}

The sensitivity of the amperometric readout circuit supports operation with a high density array of microelectrodes, whose low-level outputs might be saturated by noise if delivered off chip for measurement. To maximize sensor performance, the transducers should be directly integrated on the readout chip surface. A post-CMOS fabrication process has been developed to form a high density electrode array directly on top of the readout circuit chip. As shown in Fig. 3, the electrodes are deposited such that they make electrical connection to the top CMOS interconnect layer through openings cut in the overglass layer during CMOS fabrication. After cleaning the chips to remove surface oxides, $5 \mathrm{~nm}$ of titanium (Ti) and $100 \mathrm{~nm}$ of gold $(\mathrm{Au})$ are deposited on the surface using low temperature physical vapor deposition that does not influence circuit performance. 


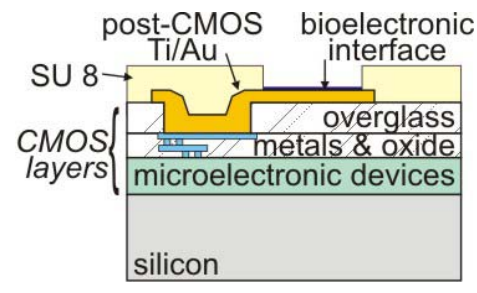

Figure 3. Illustrated profile of a post-CMOS electrode showing the Ti/Au, spin-on dielectric, and bioelectronic interface layers deposited and patterned or self assembled on a CMOS chip to transfer current from the surface electrode to on-chip integrated circuits.

Gold was chosen because it is chemically inert, has a relative high electrochemical oxidation potential, and biointerface molecules can be readily immobilized to its surface by thiol coupling [6]. After metal deposition, the Ti/Au thin films are patterned by photolithography to form an array of disk WEs and a CE that surrounds the WEs, as depicted in Fig. 2. This minimizes the resistive path for current flowing from the CE to any WE. A profile study of the chip's surface indicates that the CMOS process inherently leaves large "bumps" around the overglass openings that should be avoided as electrode sites. This can easily be achieved using the arrangement shown in Fig. 3. Although electrode-tocircuit contact points (overglass openings) are defined during CMOS fabrication, the final electrode sizes and geometries can be readily varied during post-CMOS processing.

A thin layer of SU-8 is then spun on and patterned through photolithography to define the electrode sites that will be open to solutions during bio-interface formation and sensor measurements. The SU-8 layer also covers the electrode edges to prevent current leakage around the modified electrode and corrosion of exposed Ti. It furthermore planarizes the surface to promote adhesion of subsequently applied layers that form liquid sample reservoirs, typically a PDMS stamp (Fig. 1). Once the chip is wirebonded to a package, reagents can be applied and removed in sequence into individual reservoirs to self assemble the bioelectronic sensor interfaces and perform sensor tasks. Although we have demonstrated that formation of on-chip $\mathrm{Ag} / \mathrm{AgCl} \mathrm{RE}$ can be implemented within this process flow [6], our new surface reservoir approach allows a RE to easily be inserted from the top during electrochemical testing to simplify post-CMOS processing during initial device characterization.

\section{TESTING AND RESUlts}

\section{A. Chip Implementation and Test Setup}

The circuit was fabricated in a $0.5 \mu \mathrm{m}$ CMOS process. Fig. 4 shows the $3 \times 3 \mathrm{~mm}^{2}$ die with circuit blocks labeled and surface electrodes illustrated. This version of the chip implements a $4 \times 4$ array of $100 \mu \mathrm{m}^{2}$ working electrodes, but the circuit and post-CMOS process can be scaled to much higher density, covering the entire surface of the chip. Array density is ultimately limited by fluid handling constraints to approximately 100 electrodes per chip with our existing equipment.

To characterize the circuitry, the PCB shown in Fig. 5 was constructed to interface the chip with a computer through a
National Instrument NI USB-6259 data acquisition (DAQ) system. A LabVIEW control program running on the computer sets and monitors the DC supply, analog ground, master clock signal and potentiostat control voltage, and it records the amperometric readout output and the actual potential at the RE. The LabVIEW program can plot the readout circuit output verses electrochemical cell potential to produce a cyclic voltammogram while the control voltage is swept at variable speeds.

The post-CMOS process described above was varied slightly to perform initial electrochemical testing. Rather than conducting tests within individual electrode reserviors, the PDMS stamp was removed after bio-interface formation and the chip wirebonds were coated with epoxy so that everything except the WE array and nearby regions of the CE were protected by epoxy and/or SU 8. An O-ring was then attached to the package, as shown in Fig. 6, to form a single liquid reservoir around the chip that holds test samples.

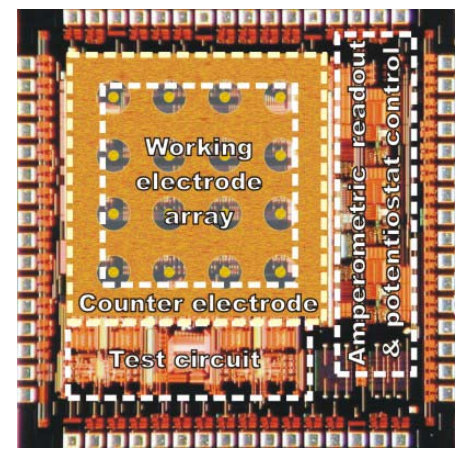

Figure 4. Die photograph of the $3 \times 3 \mathrm{~mm}$ CMOS chip with surface electrodes and circuit blocks labeled.

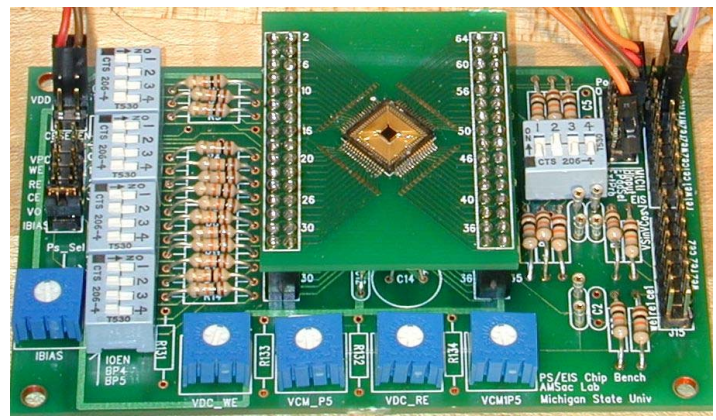

Figure 5. Testing interface for the bioelectrochemical sensor readout and electrode array chip (center). The board is connected to a DAQ and a PC running LabVIEW.

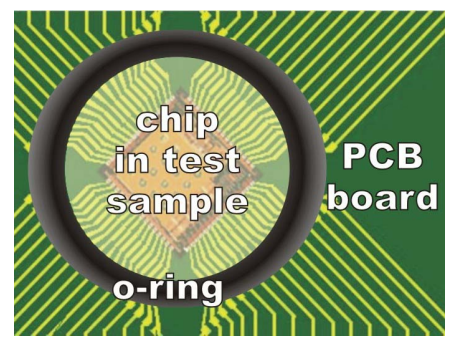

Figure 6. Close-up image of the electrochemcial test setup with an O-ring to contain liquid samples. 


\section{B. Test Results}

A triangle waveform control signal was applied to the onchip potentiostat, and the resulting RE-to-WE potential was measured. The voltage was shown to properly transfer to the on-chip electrochemical cell, demonstrating that cyclic voltammetry can be performed with on-chip electronics. The readout circuit was verified to measure input currents ranging from as low as $10 \mathrm{pA}$ to as high as $10 \mu \mathrm{A}$, with high linearity over this input span. Noise analysis indicated the resolution was below $1 \mathrm{pA}$.

To verify that the on-chip electrochemical interface circuit properly performs cyclic voltammetry measurements, $\mathrm{K}_{3} \mathrm{Fe}(\mathrm{CN})_{6}$ (potassium ferricyanide, J.T.Baker) was used as an analyte. The $\mathrm{Fe}^{\mathrm{III}}(\mathrm{CN})_{6}^{3-} / \mathrm{Fe}^{\mathrm{II}}(\mathrm{CN})_{6}{ }^{4-}$ couple is known to be chemically and electrochemically reversible. $1 \mathrm{mM} \mathrm{K}_{3} \mathrm{Fe}(\mathrm{CN})_{6}$ and $10 \mathrm{mM} \mathrm{KCl}$ (potassium chloride, J.T. Baker) were added to a beaker with a funnel-shaped bottom to minimize convection. An Omni EE006 1mm diameter gold electrode (Cypress Systems), which is sealed in a glass cylinder, was utilized as the working electrode. An Omni EE006 platinum electrode (Cypress Systems) was used the as counter electrode. The reference electrode was an Accumet gel-filled epoxybody calomel electrode (Fisher Scientific) with $0.245 \mathrm{~V}$ vs. NHE. The RE was stored in saturated $\mathrm{KCl}$ and calibrated before use.

The supply voltage and analog ground were set to $3.3 \mathrm{~V}$ and $1.6 \mathrm{~V} \mathrm{DC}$, respectively. A $530 \mathrm{mV}$ peak-to-peak triangle wave was generated and applied between $\mathrm{CE}$ and WE, sweeping from $-100 \mathrm{mV}$ to $430 \mathrm{mV}$ (with respect to the RE potential) at $2.5 \mathrm{~Hz}$. The on-chip amperometric readout circuit was clocked at $800 \mathrm{kHz}$, and the cyclic voltammogram shown in Fig. 7 was captured. The typical shape of this curve verifies the on-chip potentiostat operates as expected and can perform cyclic voltammetry. Although the circuit measures current from the selected electrochemical cell, the amplified output was recorded as a voltage. The data can be translated back to current using (1) once precise characterization of on-chip capacitances has been performed. This will allow a direct comparison with commercial equipment. Now that the chip has been proven reliable for basic electrochemical measurements, bioelectronic interfaces can be applied to the electrodes and assessed using the on-chip bioelectrochemical readout circuit.

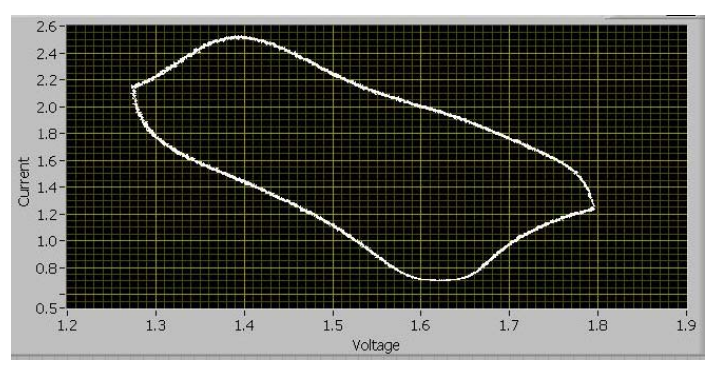

Figure 7. Cyclic voltammogram measured by the on-chip poteniostat and recorded on a computer through a DAQ card.

\section{CONCLUSION}

A single-chip amperometric readout circuit and electrode array system suitable for bioelectrochemical measurements has been designed and implemented. The on-chip circuitry features an electrode potential control block and a current readout block that serve as an electrochemical potentiostat capable of performing chronoamperometery and cyclic voltammetry. The readout signal range and sensitivity and the potential sweep capability are tailored to the needs of nanostructured, protein-based, bioelectronic interfaces. Measurements show the chip can perform amperometric readout over a $10 \mathrm{pA}$ to $10 \mu \mathrm{A}$ range and can resolve sub-pA currents from a multiplexed array of on-chip working electrodes. A post-CMOS fabrication process suitable for forming an array of electrochemical electrodes on the surface of the chip was also described. The defined structures and process sequence overcome many pitfalls to interfacing organic materials with CMOS circuits. A $4 \times 4$ array of working electrodes was initially implemented on the surface of the $3 \times 3 \mathrm{~mm}^{2}$ readout chip using techniques that can readily be scaled to an array of 100 or more electrodes.

\section{REFERENCES}

[1] M. I. Prodromidis and M. I. Karayannis, "Enzyme based amperometric Biosensors for food analysis," Electroanalysis, vol. 14, pp. 241-261, Mar, 2002.

[2] B. L. Hassler and R. M. Worden, "Versatile bioelectronic interfaces based on heterotrifunctional linking molecules," Biosensors \& Bioelectronics, vol. 21, pp. 2146-2154, May, 2006.

[3] S. F. Peteu, D. Emerson, and R. M. Worden, "A Clark-type oxidase enzyme-based amperometric microbiosensor for sensing glucose, galactose, or choline," Biosensors \& Bioelectronics, vol. 11, pp. 10591071, 1996.

[4] S. F. Peteu, M. T. Widman, and R. M. Worden, "In situ mapping of community-level cellular response with catalytic microbiosensors," Biosensors \& Bioelectronics, vol. 13, pp. 1197-1203, Nov, 1998.

[5] J. Zhang, N. Trombly, and A. Mason, "A Low Noise Readout Circuit for Integrated Electrochemical Biosensor Arrays," IEEE Int. Conf. on Sensors, Vienna, Austria, pp. 36-39, October, 2004.

[6] R. M. Worden, B. Hassler, P. Kim, N. Kohli, A. Mason, J. G. Zeikus, M. Laivenieks, and R. Ofoli, "Biomimetic Interfaces for a Multifunctional Biosensor Array Microsystem," IEEE Int. Conf. on Sensors, Vienna, Austria, pp. 991-994, Oct., 2004.

[7] R. G. Kakerow, H. Kappert, E. Spiegel, and Y. Manoli, "Low-Power Single-Chip CMOS Potentiostat," Int. Conf. Solid-State Sensors and Actuators (Transducers), vol. 1, pp. 142-145, June, 1995.

[8] M. Breten, T. Lehmann, and E. Braun, "Integrating data converters for picoampere currents from electrochemical transducers," IEEE International Symposium on Circuits and Systems, Geneva, pp. 709712,2000

[9] H. S. Narula and J. G. Harris, "VLSI Potentiostat for Amperometric Measurements for Electrolytic Reactions," IEEE International Symposium Circuits and Systems, Vancouver, Canada, pp. 457-460, May, 2004.

[10] M. Schienle, C. Paulus, A. Frey, F. Hofmann, B. Holzapfl, P. Schindler-Bauer, and R. Thewes, "A Fully Electronic DNA Sensor with 128 Positions and In-Pixel A/D Conversion," J. Solid-State Circuits, vol. 39, pp. 2438-2445, December, 2004.

[11] C. C. Enz and G. C. Temes, "Circuit techniques for reducing the effects of op-amp imperfections: autozeroing, correlated double sampling, and chopper stabilization," Proceedings of the IEEE, vol. 84, pp. 1584$1614,1996$. 